\title{
Phosphaquinomethanes and Phosphathienoquinomethanes, and Their Anion Radicals
}

\author{
Fumiki Murakami, Shigeru Sasaki, and Masaaki Yoshifuji* \\ Department of Chemistry, Graduate School of Science, Tohoku University, \\ Aoba, Sendai 980-8578, Japan \\ yoshifj@mail.tains.tohoku.ac.jp
}

Experimental Section

\section{General.}

${ }^{1} \mathrm{H},{ }^{13} \mathrm{C}$, and ${ }^{31} \mathrm{P}$ NMR spectra were measured on a Bruker AC200P, AV400, AM600, or JEOL JNM-A500 spectrometer. ${ }^{1} \mathrm{H}$ and ${ }^{13} \mathrm{C}$ NMR chemical shifts are expressed as $\delta$ downfield from external tetramethylsilane. ${ }^{31} \mathrm{P}$ NMR chemical shifts are expressed as $\delta$ downfield from $85 \% \mathrm{H}_{3} \mathrm{PO}_{4}$. Infrared spectra and ultraviolet-visible spectra were collected on a Horiba FT-300 spectrometer and a Hitachi U-3210 spectrophotometer, respectively. Mass spectra were measured on a Hitachi M-2500 with electron impact (EI) ionization at $70 \mathrm{eV}$ or a JEOL HX-110 with fast atom bombardment (FAB) ionization using $m$-nitrobenzyl alcohol matrix. Melting points were measured on a Yanagimoto MP-J3 apparatus without correction. Microanalyses were performed at the Instrumental Analysis Center of Chemistry, Graduate School of Science, Tohoku University. Merck silica gel (Kieselgel 60) and Sumitomo basic alumina (KCG-30) were used for column chromatography. All reactions were carried out under argon. Tetrahydrofuran and diethyl ether were distilled from sodium benzophenone ketyls under argon just prior to use.

[4-(diphenylhydroxylmethyl)phenyl](2,4,6-tri-tert-butylphenyl)phosphine (9)

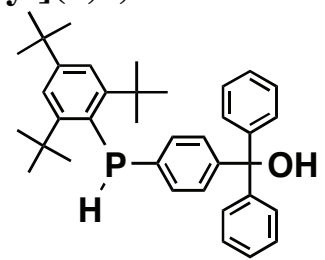

To a solution of 4-bromophenyl(2,4,6-tri-tert-butylphenyl)phosphine (7) (2.00 g, $4.62 \mathrm{mmol})$ in ether $(20 \mathrm{~mL})$ was added $t$-butyllithium $(6.0 \mathrm{~mL}, 1.54 \mathrm{M}$ in pentane $)$ at $78{ }^{\circ} \mathrm{C}$. After being stirred for 45 minutes at $-78{ }^{\circ} \mathrm{C}$, the resultant orange solution of 4$\{(2,4,6$-tri-tert-butylphenyl)phosphino $\}$ phenyllithium was added over $10 \mathrm{~min}$ to a solution of benzophenone $(813 \mathrm{mg}, 4.46 \mathrm{mmol})$ in ether $(20 \mathrm{~mL})$ at $-78{ }^{\circ} \mathrm{C}$. After being stirred for $1 \mathrm{~h}$ at $-78{ }^{\circ} \mathrm{C}$, the deep green solution was stirred for $10 \mathrm{~h}$ at room temperature. The resultant solution was washed with dilute $\mathrm{NH}_{4} \mathrm{Cl}$ solution and saturated $\mathrm{NaCl}$ solution. The organic layer was dried over anhydrous magnesium sulfate. After removal of the drying agent and the solvent, the residue was submitted to column chromatography (silica gel, hexane to hexane/ethyl acetate $(50: 1, \mathrm{v} / \mathrm{v})$ to give $2.06 \mathrm{~g}(3.84 \mathrm{mmol}, 86 \%)$ of 9. 9 : colorless plates $(\mathrm{EtOH}) ; \mathrm{mp} 150-154{ }^{\circ} \mathrm{C}(\mathrm{dec}) ;{ }^{1} \mathrm{H} \mathrm{NMR}\left(200 \mathrm{MHz}, \mathrm{CDCl}_{3}\right) \delta=7.47(2 \mathrm{H}$, $\left.\mathrm{d}, J_{\mathrm{PH}}=2.3 \mathrm{~Hz}, \mathrm{Mes}^{*}-m\right), 7.26(6 \mathrm{H}, \mathrm{m}, \mathrm{Ph}-m, p), 7.18(6 \mathrm{H}, \mathrm{m}, \mathrm{Ph}-o), 7.00\left(2 \mathrm{H}, \mathrm{d}, J_{\mathrm{HH}}=7.1\right.$ $\mathrm{Hz}), 6.64\left(2 \mathrm{H}, \mathrm{d}, J_{\mathrm{HH}}=7.2 \mathrm{~Hz}\right), 6.04\left(1 \mathrm{H}, \mathrm{d}, J_{\mathrm{PC}}=218 \mathrm{~Hz},-\mathrm{PH}\right), 2.7(1 \mathrm{H}, \mathrm{brs},-\mathrm{OH}), 1.46$ $\left(18 \mathrm{H}, \mathrm{s}, \mathrm{C}\left(\mathrm{CH}_{3}\right)_{3}-o\right), 1.33\left(9 \mathrm{H}, \mathrm{s}, \mathrm{C}\left(\mathrm{CH}_{3}\right)_{3}-p\right) ;{ }^{13} \mathrm{C} \mathrm{NMR}\left(100 \mathrm{MHz}, \mathrm{CDCl}_{3}\right) \delta=155.85$ (brs, Mes*-o), 150.40 (Mes*-p), 146.78 (s, Ph-i), 145.34 (s, $\left.\mathrm{C}_{6} \mathrm{H}_{4}-p\right), 139.54$ (d, $J=14.68 \mathrm{~Hz}$, $\left.\mathrm{C}_{6} \mathrm{H}_{4}-i\right), 130.54$ (d, $J=16.69 \mathrm{~Hz}, \mathrm{C}_{6} \mathrm{H}_{4}-o$ ), 127.82 (s, Ph-o, $m$ ), 127.76 (d, $J=25.68 \mathrm{~Hz}$, Mes*-i), 127.52 (d, $\left.J=5.62 \mathrm{~Hz}, \mathrm{C}_{6} \mathrm{H}_{4}-m\right), 127.16$ (s, Ph- $p$ ), 122.30 (d, $J=3.75 \mathrm{~Hz}, \mathrm{Mes}_{-}{ }_{-}$ 
m), 81.84 (s, COH), 38.32 (brs, $C \mathrm{Me}_{3}-o$ ), 35.08 (s, $\left.C \mathrm{Me}_{3}-p\right), 33.45$ (d, $J=6.82 \mathrm{~Hz}$, $\left.\mathrm{C}\left(\mathrm{CH}_{3}\right)_{3}-o\right), 31.30\left(\mathrm{~s}, \mathrm{C}\left(\mathrm{CH}_{3}\right)_{3}-p\right) ;{ }^{31} \mathrm{P} \mathrm{NMR}\left(81 \mathrm{MHz}, \mathrm{CDCl}_{3}\right) \delta=-65.0\left(\mathrm{~d}, J_{\mathrm{PH}}=231 \mathrm{~Hz}\right)$; IR(KBr) 3498(brm), 2960(s), 2918(m), 2870(m), 2393(m), 1917(w), 1647(m), 1601(m), 1508(m), 1460(m), 1398(m), 1362(m), 1315(w), 1281(w), 1207(w), 1184(m) 1109(w), 1018(m), 920(m), 877(w), 814(w), 750(m), 728(w), 611(w), 579(m), 525(w), 490(w), and 467(w) $\mathrm{cm}^{-1}$; LRMS (70 eV, EI) $\mathrm{m} / z$ (rel intensity) $536\left(M^{+} ; 75\right), 535\left(M^{+}-\mathrm{H} ; 100\right), 519$ $\left(M^{+}-\mathrm{OH} ; 7\right), 480\left(M^{+}-t-\mathrm{Bu}+\mathrm{H} ; 5\right), 263\left(\mathrm{Mes}^{*} \mathrm{PH}_{2}-\mathrm{CH}_{3} ; 21\right), 231\left(\mathrm{Mes}^{*}-\mathrm{CH}_{3}+\mathrm{H} ; 15\right)$; Found: C, 81.669; H, 8.274 \%. Calcd for $\mathrm{C}_{37} \mathrm{H}_{45} \mathrm{OP}: \mathrm{C}, 82.798 ; \mathrm{H}, 8.451 \%$.

\section{Phosphaquinomethane 5}

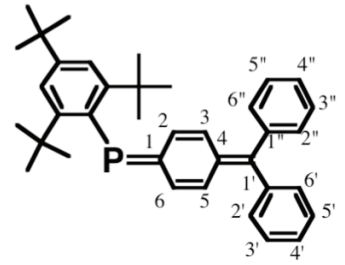

$\mathrm{CF}_{3} \mathrm{COOH}(0.03 \mathrm{~mL}, 0.389 \mathrm{mmol})$ was added to a solution of carbinol 9 (200 $\mathrm{mg}$, $0.373 \mathrm{mmol})$ in dry benzene $(5 \mathrm{~mL})$ at room temperature and the mixture was stirred for 10 min. Triethylamine $(0.3 \mathrm{~mL})$ was then added at room temperature. The reaction mixture was submitted to column chromatography (Alumina, benzene) to give $173 \mathrm{mg}$ $(0.333 \mathrm{mmol}, 89 \%)$ of 5. 5: orange crystals, mp 79.0-82.0 ${ }^{\circ} \mathrm{C}$ (Decomp.); ${ }^{1} \mathrm{H}$ NMR (600 $\left.\mathrm{MHz}, \mathrm{CD}_{2} \mathrm{Cl}_{2}\right) \delta=7.44\left(2 \mathrm{H}, \mathrm{s}, \mathrm{Mes}^{*}-m\right), 7.34-7.24$ (6H, m, arom.-3',4',5',3",4",5"), 7.21 $\left(2 \mathrm{H}, \mathrm{dd}, J_{\mathrm{HH}}=1.2,3.4 \mathrm{~Hz}\right.$, arom.-2',6' or 2",6"), $7.17\left(2 \mathrm{H}, \mathrm{dd}, J_{\mathrm{HH}}=2.0,6.6 \mathrm{~Hz}\right.$, arom.2',6' or 2",6"), $7.11\left(1 \mathrm{H}\right.$, ddd, $J_{\mathrm{HH}}=1.7,4.1 \mathrm{~Hz}, J_{\mathrm{PH}}=13.8 \mathrm{~Hz}$, quinone-6), $6.78(1 \mathrm{H}$, ddd, $J_{\mathrm{HH}}=1.7,4.5 \mathrm{~Hz}, J_{\mathrm{PH}}=9.7 \mathrm{~Hz}$, quinone-5), $6.46\left(1 \mathrm{H}, \mathrm{ddd}, J_{\mathrm{HH}}=1.7, J_{\mathrm{HH}}=5.6 \mathrm{~Hz}, J_{\mathrm{PH}}=\right.$ $9.6 \mathrm{~Hz}$, quinone-3), $5.48\left(1 \mathrm{H}, \mathrm{ddd}, J_{\mathrm{HH}}=1.6, J_{\mathrm{HH}}=6.2 \mathrm{~Hz}, J_{\mathrm{PH}}=9.6 \mathrm{~Hz}\right.$, quinone-2), 1.46 $\left(18 \mathrm{H}, \mathrm{s}, \mathrm{C}\left(\mathrm{C}_{3}\right)_{3}-o\right), 1.33\left(9 \mathrm{H}, \mathrm{s}, \mathrm{C}\left(\mathrm{C}_{3}\right)_{3}-p\right),{ }^{13} \mathrm{C}\left\{{ }^{1} \mathrm{H}\right\} \mathrm{NMR}\left(151 \mathrm{MHz}, \mathrm{CD}_{2} \mathrm{Cl}_{2}\right) \delta=$ $170.35\left(\mathrm{~d}, J_{\mathrm{PC}}=29.1 \mathrm{~Hz}, \mathrm{P}=\underline{\mathrm{C}}-1\right), 155.85\left(\mathrm{~s}, \mathrm{Mes}^{*}-o\right), 151.03\left(\mathrm{~s}, \mathrm{Mes}^{*}-p\right), 142.82\left(\mathrm{~d}, J_{\mathrm{PC}}=\right.$ $10.7 \mathrm{~Hz}, \mathrm{C}-1^{\prime}$ ), 142.78 (d, $\left.J_{\mathrm{PC}}=10.7 \mathrm{~Hz}, \mathrm{C}^{\prime} 1^{\prime}\right), 142.43$ (d, $\left.J_{\mathrm{PC}}=52.2 \mathrm{~Hz}, C-4=\mathrm{C}\right), 136.47$ $\left(\mathrm{d}, J_{\mathrm{PC}}=53.1 \mathrm{~Hz}\right.$, Mes$^{*}$-ipso $), 134.68\left(\mathrm{~d}, J_{\mathrm{PC}}=28.5 \mathrm{~Hz}, \mathrm{C}-4=C\right), 133.19\left(\mathrm{~d}, J_{\mathrm{PC}}=30.3 \mathrm{~Hz}\right.$, C-6), 131.96 (d, $\left.J_{\mathrm{PC}}=9.4 \mathrm{~Hz}, \mathrm{C}^{\prime} 2^{\prime}, 6^{\prime}\right), 131.92\left(\mathrm{~d}, J_{\mathrm{PC}}=9.2 \mathrm{~Hz}, \mathrm{C}-2^{\prime}, 6^{\prime}\right), 128.98\left(\mathrm{~d}, J_{\mathrm{PC}}=\right.$ $20.4 \mathrm{~Hz}, \mathrm{C}-2$ ), 128.82 (s, C-3',5'), 128.77 (s, C-3',5'), 128.42 (s, C-4'), 128.33 (s, C-4'), $126.97\left(\mathrm{~d}, J_{\mathrm{PC}}=29.7 \mathrm{~Hz}, \mathrm{C}-3\right), 126.13\left(\mathrm{~d}, J_{\mathrm{PC}}=43.0 \mathrm{~Hz}, \mathrm{C}-5\right), 122.42(\mathrm{~s}, \mathrm{Mes} *-m), 38.843$ (s, $\left.\underline{\mathrm{C}}\left(\mathrm{CH}_{3}\right)_{3}-o\right), 35.707\left(\mathrm{~s}, \underline{\mathrm{C}}\left(\mathrm{CH}_{3}\right)_{3}-p\right), 33.775\left(\mathrm{~d}, J_{\mathrm{PC}}=5.1 \mathrm{~Hz}, \mathrm{C}\left(\underline{\mathrm{C}} \mathrm{H}_{3}\right)_{3}-o\right), 31.867$ (s, $\left.\mathrm{C}\left(\mathrm{CH}_{3}\right)_{3}-p\right) ;{ }^{31} \mathrm{P}$ NMR $\left(81 \mathrm{MHz}, \mathrm{C}_{6} \mathrm{D}_{6}\right) \delta=244.2$ (s); ${ }^{31} \mathrm{P} \mathrm{NMR}\left(81 \mathrm{MHz}, \mathrm{CD}_{2} \mathrm{Cl}_{2}\right) \delta=$ $244.4(\mathrm{~s}) ; \mathrm{UV}-\mathrm{Vis}$ (hexanes) $\lambda_{\max } / \mathrm{nm}(\log \varepsilon) 440$ (4.49); IR(KBr) 2960(s), 2908(m), 2868(m), 1662(w), 1630(w), 1593(m), 1525(w), 1479(m), 1448(m), 1394(m), 1362(m), 1279(w), 1240(m), 1211(m), 1184(m), 1122(w), 1028(w), 930(w), 825(w), 760(m), 729(w), 700(m), 540(w), 490(w), and 444(w) $\mathrm{cm}^{-1}$; LRMS (70 eV, EI) $\mathrm{m} / \mathrm{z}$ (rel intensity) $518\left(M^{+} ; 100\right), 503\left(M^{+}-\mathrm{CH}_{3} ; 11\right), 476\left(M^{+}-\mathrm{CMe}_{2} ; 29\right), 261$ (Mes*P-CH $\left.; 28\right), 220$ (Mes*P- $t$-Bu+H; 11), $86(t-\mathrm{BuP}-2 \mathrm{H} ; 13), 57(t-\mathrm{Bu}, 11)$; HRMS (70 eV, EI) Found $m / z$ 518.2728. Calcd. for $\mathrm{C}_{37} \mathrm{H}_{43} \mathrm{P}: M, 518.3102$

\section{Phosphaquinomethane $5-{ }^{13} \mathrm{C}$}

${ }^{13} \mathrm{C}$-labeled phosphaquinomethane $5-{ }^{13} \mathrm{C}$ was synthesized by the procedure similar to 5. Benzophenone-carbonyl $-{ }^{13} \mathrm{C}$ (Aldrich 99 atom $\%{ }^{13} \mathrm{C}$ ) was used in place of benzophenone. $\quad \mathbf{9 -}^{13} \mathrm{C}:{ }^{31} \mathrm{P} \mathrm{NMR}\left(81 \mathrm{MHz}, \mathrm{CDCl}_{3}\right) \delta=-65.0\left(\mathrm{~d}, J_{\mathrm{PH}}=231 \mathrm{~Hz}\right),{ }^{13} \mathrm{C} \mathrm{NMR}$ $\left(50 \mathrm{MHz}, \mathrm{CDCl}_{3}\right) \delta=81.8(\mathrm{~s}) . \quad \mathbf{5 -}^{13} \mathrm{C}:{ }^{31} \mathrm{P} \mathrm{NMR}\left(81 \mathrm{MHz}, \mathrm{C}_{6} \mathrm{D}_{6}\right) \delta=244.2\left(\mathrm{~d}, J_{\mathrm{PC}}=28.5\right.$ $\mathrm{Hz}),{ }^{13} \mathrm{C}$ NMR $\left(50 \mathrm{MHz}, \mathrm{C}_{6} \mathrm{D}_{6}\right) \delta=134.7\left(\mathrm{~d}, J_{\mathrm{PC}}=28.5 \mathrm{~Hz}, \mathrm{C}-4=C\right)$.

\section{Phosphathienoquinomethane (Z)-6a}




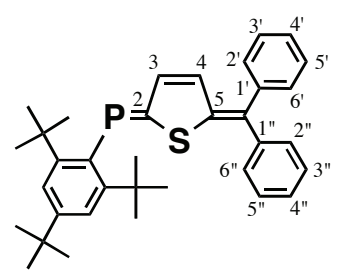

To a solution of 5-bromo-2-thienyl(2,4,6-tri-tert-butylphenyl)phosphine (8) (362 $\mathrm{mg}, 0.825 \mathrm{mmol})$ in ether $(20 \mathrm{~mL})$ was added $t$-butyllithium $(1.0 \mathrm{~mL}, 1.64 \mathrm{M}$ in pentane) at $-78{ }^{\circ} \mathrm{C}$ and stirred for $30 \mathrm{~min}$. The resultant pale orange solution was added to benzophenone $(160 \mathrm{mg}, 0.873 \mathrm{mmol})$ in ether $(10 \mathrm{~mL})$ at $-78^{\circ} \mathrm{C}$ and stirred for $30 \mathrm{~min}$. The mixture was gradually warmed to room temperature and stirred for $12 \mathrm{~h}$. The solution was washed with dilute $\mathrm{NH}_{4} \mathrm{Cl}$ solution and water. The organic layer was dried over anhydrous magnesium sulfate. After removal the drying agent and the solvent, formation of the mixture of carbinol 10a and (Z)-6a was confirmed by ${ }^{31} \mathrm{P}$ NMR spectroscopy. The mixture of 10a and (Z)-6a was dissolved in dry benzene (15 mL), added to a suspension of anhydrous copper(II) sulfate $(1.29 \mathrm{~g}, 8.10 \mathrm{mmol})$ in dry benzene $(10 \mathrm{~mL})$ at room temperature, and refluxed for $3 \mathrm{~h}$. Consumption of the starting material and formation of (Z)-6a were monitored by ${ }^{31} \mathrm{P}$ NMR spectroscopy. The reaction mixture was filtered to remove copper sulfate. After removal of the drying agent and the solvent, the residue was submitted to column chromatography (alumina, benzene) to give $392 \mathrm{mg}(0.747 \mathrm{mmol}, 90 \%)$ of $(Z)-6 a . \quad$ 10a: ${ }^{31} \mathrm{P} \mathrm{NMR}\left(81 \mathrm{MHz}, \mathrm{C}_{6} \mathrm{D}_{6}\right) \delta=-76.5(\mathrm{~d}$, $J_{\mathrm{PH}}=233 \mathrm{~Hz}$ ). (Z)-6a; orange crystals, mp 120.0-122.0 ${ }^{\circ} \mathrm{C}$ (Decomp.); ${ }^{1} \mathrm{H}$ NMR (600 $\left.\mathrm{MHz}, \mathrm{CD}_{2} \mathrm{Cl}_{2}\right) \delta=7.32\left(2 \mathrm{H}, \mathrm{d}, J_{\mathrm{PH}}=1.1 \mathrm{~Hz}, \mathrm{Mes}^{*}-m\right), 7.24-7.20$ (4H, m, arom.2',6’,2”,6”), 7.10-7.06 (6H, m, arom.-3',4',5',3”,4”, 5”), $6.96\left(1 \mathrm{H}, \mathrm{dd}, J_{\mathrm{HH}}=5.8 \mathrm{~Hz}, J_{\mathrm{PH}}=\right.$ $8.1 \mathrm{~Hz}$, quinone-3), $6.72\left(1 \mathrm{H}, \mathrm{dd}, J_{\mathrm{HH}}=5.8 \mathrm{~Hz}, J_{\mathrm{PH}}=4.2 \mathrm{~Hz}\right.$, quinone-4), $1.42(18 \mathrm{H}, \mathrm{s}$, $\left.\mathrm{C}\left(\mathrm{C}_{3}\right)_{3}-o\right), 1.21\left(9 \mathrm{H}, \mathrm{s}, \mathrm{C}\left(\mathrm{C}_{3}\right)_{3}-p\right),{ }^{13} \mathrm{C}\left\{{ }^{1} \mathrm{H}\right\} \mathrm{NMR}\left(151 \mathrm{MHz}, \mathrm{CD}_{2} \mathrm{Cl}_{2}\right) \delta=182.92\left(\mathrm{~d}, J_{\mathrm{PC}}\right.$ $=47.9 \mathrm{~Hz}, \mathrm{P}=\mathrm{C}), 154.75\left(\mathrm{~s}, \mathrm{Mes}^{*}-o\right), 151.40\left(\mathrm{~s}, \mathrm{Mes}^{*}-p\right), 146.26\left(\mathrm{~d}, J_{\mathrm{PC}}=20.6 \mathrm{~Hz}, \mathrm{C}-5\right)$, $142.73\left(\mathrm{~d}, J_{\mathrm{PC}}=7.1 \mathrm{~Hz}, \mathrm{C}-1\right.$ ”), $142.32\left(\mathrm{~d}, J_{\mathrm{PC}}=6.2 \mathrm{~Hz}, \mathrm{C}-1\right.$ '), $139.26\left(\mathrm{~d}, J_{\mathrm{PC}}=49.4 \mathrm{~Hz}\right.$, Mes*-ipso), $138.53\left(\mathrm{~d}, J_{\mathrm{PC}}=21.5 \mathrm{~Hz}, \mathrm{C}-3\right), 133.85\left(\mathrm{~d}, J_{\mathrm{PC}}=33.4 \mathrm{~Hz}, \mathrm{C}-4\right), 131.41\left(\mathrm{~d}, J_{\mathrm{PC}}=\right.$ $3.5 \mathrm{~Hz}, \mathrm{C}-2$ ',6'), 130.44 (d, $\left.J_{\mathrm{PC}}=35.2 \mathrm{~Hz}, \mathrm{C}-6\right), 130.45$ (d, $J_{\mathrm{PC}}=3.5 \mathrm{~Hz}, \mathrm{C}-2$ "”, 6”), 128.93 (s, C-3', 5'), 128.78 (s, C-3”, 5”), 128.18 (s, C-4', 4”), 122.81 (s, Mes*-m), 38.70 (s, $\left.\mathrm{C}\left(\mathrm{CH}_{3}\right)_{3}-o\right), 35.68\left(\mathrm{~s}, \underline{\mathrm{C}}\left(\mathrm{CH}_{3}\right)_{3}-p\right), 33.21\left(\mathrm{~d}, J_{\mathrm{PC}}=6.2 \mathrm{~Hz}, \mathrm{C}\left(\mathrm{CH}_{3}\right)_{3}-o\right), 31.87\left(\mathrm{~s}, \mathrm{C}\left(\mathrm{CH}_{3}\right)_{3}-p\right)$; ${ }^{31} \mathrm{P}$ NMR (81 MHz, $\left.\mathrm{CD}_{2} \mathrm{Cl}_{2}\right) \quad \delta=201.4(\mathrm{~s}) ;{ }^{31} \mathrm{P} \mathrm{NMR}\left(81 \mathrm{MHz}, \mathrm{C}_{6} \mathrm{D}_{6}\right) \quad \delta=201.5(\mathrm{~s})$; IR(KBr) 2960(s), 2908(m), 2868(m), 1662(w), 1630(w), 1593(m), 1525(w), 1479(m), 1448(m), 1394(m), 1362(m), 1279(w), 1240(m), 1211(m), 1184(m), 1122(w), 1028(w), 930(w), 825(w), 760(m), 729(w), 700(m), 540(w), 490(w), and 444(w) $\mathrm{cm}^{-1}$; UV-Vis (hexanes) $\lambda_{\max } / \mathrm{nm}(\log \varepsilon) 446$ (4.49), 312 (sh; 3.70), 282 (3.99); LRMS (70 eV, EI) $\mathrm{m} / \mathrm{z}$ (rel intensity) $524\left(M^{+} ; 100\right)$; HRMS (70 eV, EI) Found $m / z$ 524.2532. Calcd. for $\mathrm{C}_{35} \mathrm{H}_{41} \mathrm{PS}$ : $\mathrm{M}$, 524.2667. (E)-3aa; ${ }^{31} \mathrm{P} \mathrm{NMR}\left(81 \mathrm{MHz}, \mathrm{C}_{6} \mathrm{D}_{6}\right) \delta=191.3$ (s)

Phosphaquinomethane (Z)-6a- ${ }^{13} \mathrm{C}$

${ }^{13} \mathrm{C}$-labeled phosphathienoquinomethane $(Z)-6 \mathbf{a}^{-13} \mathrm{C}$ was synthesized by the procedure similar to $(Z)$-6a. Benzophenone-carbonyl $-{ }^{13} \mathrm{C}$ (Aldrich 99 atom $\%{ }^{13} \mathrm{C}$ ) was used in place of benzophenone. $(Z)-6 \mathbf{a}^{13}{ }^{13} \mathrm{C}: \quad{ }^{31} \mathrm{P} \mathrm{NMR}\left(81 \mathrm{MHz}, \mathrm{C}_{6} \mathrm{D}_{6}\right) \delta=201.5(\mathrm{~d}$, $\left.J_{\mathrm{PC}}=35.5 \mathrm{~Hz}\right) ;{ }^{13} \mathrm{C}$ NMR $\left(50 \mathrm{MHz}, \mathrm{C}_{6} \mathrm{D}_{6}\right) \delta=129.48\left(\mathrm{~d}, J_{\mathrm{PC}}=35.5 \mathrm{~Hz}\right) . \quad(E)-6 \mathbf{6}{ }^{13}{ }^{13}$ :

${ }^{31} \mathrm{P}$ NMR $\left(81 \mathrm{MHz}, \mathrm{C}_{6} \mathrm{D}_{6}\right) \delta=191.3\left(\mathrm{~d}, J_{\mathrm{PC}}=36.9 \mathrm{~Hz}\right) . \quad \mathbf{1 0 a}-{ }^{13} \mathbf{C}:{ }^{31} \mathrm{P} \quad \mathrm{NMR} \quad(81$ $\left.\mathrm{MHz}, \mathrm{C}_{6} \mathrm{D}_{6}\right) \delta=-76.6\left(\mathrm{~d}, J_{\mathrm{PH}}=233 \mathrm{~Hz}\right),{ }^{13} \mathrm{C} \mathrm{NMR}\left(50 \mathrm{MHz}, \mathrm{C}_{6} \mathrm{D}_{6}\right) \delta=78.9$.

Phosphathienoquinomethane $(Z)-6 b$ 


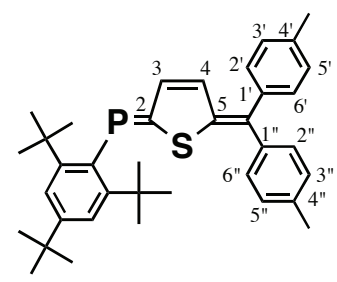

5-Bromo-2-thienyl(2,4,6-tri-tert-butylphenyl)phosphine (8) (440 mg, $1.00 \mathrm{mmol})$ in ether $(10 \mathrm{~mL})$ was added $t$-butyllithium $(1.5 \mathrm{~m}, 1.45 \mathrm{M}$ in pentane $)$ at $-78{ }^{\circ} \mathrm{C}$ and stirred for $30 \mathrm{~min}$. The resultant orange solution was added over $5 \mathrm{~min}$ to 4,4'dimethylbenzophenone $(227 \mathrm{mg}, 1.08 \mathrm{mmol})$ in ether $(15 \mathrm{~mL})$ at $-78{ }^{\circ} \mathrm{C}$ and stirred for 30 min. The mixture was gradually warmed to room temperature and stirred for $10 \mathrm{~h}$. The solution was washed with dilute $\mathrm{NH}_{4} \mathrm{Cl}$ solution and water. The organic layer was dried over anhydrous magnesium sulfate. After removal of the drying agent and the solvent, formation of a mixture of carbinol $\mathbf{1 0 b}$ and (Z)-6b was confirmed by ${ }^{31} \mathrm{P}$ NMR spectroscopy. The mixture of $\mathbf{1 0 b}$ and $(Z)-\mathbf{6 b}$ was dissolved in dry benzene $(15 \mathrm{~mL})$, added to a suspension of anhydrous copper(II) sulfate $(1.69 \mathrm{~g}, 8.69 \mathrm{mmol})$ in dry benzene $(10 \mathrm{~mL})$ at room temperature, and refluxed for $3 \mathrm{~h}$. Consumption of $\mathbf{1 0 b}$ and formation of $(Z)-\mathbf{6 b}$ were monitored by ${ }^{31} \mathrm{P}$ NMR spectroscopy. The reaction mixture was filtered to remove copper sulfate. After removal of the drying agent and the solvent, the residue was submitted to column chromatography (alumina, benzene) to give $500 \mathrm{mg}(0.905 \mathrm{mmol}$, $90 \%)$ of (Z)-6b. 10b: ${ }^{31} \mathrm{P}$ NMR $\left(81 \mathrm{MHz}, \mathrm{C}_{6} \mathrm{D}_{6}\right) \delta=-76.4\left(\mathrm{~d}, J_{\mathrm{PH}}=232 \mathrm{~Hz}\right) . \quad(Z)-\mathbf{6 b}$ : orange prisms (Ether), mp 162.0-163.0 ${ }^{\circ} \mathrm{C}$ (dec.); ${ }^{1} \mathrm{H}$ NMR $\left(500 \mathrm{MHz}, \mathrm{CD}_{2} \mathrm{Cl}_{2}\right) \delta=7.36$ $\left(2 \mathrm{H}, \mathrm{d},{ }^{4} J_{\mathrm{PH}}=1.0 \mathrm{~Hz}, \mathrm{Mes}^{*}-m\right), 7.08\left(2 \mathrm{H}, \mathrm{d},{ }^{3} J_{\mathrm{HH}}=8.2 \mathrm{~Hz}\right.$, arom.-3', 5'), $7.01\left(2 \mathrm{H}, \mathrm{d},{ }^{3} J_{\mathrm{HH}}\right.$ $=8.2 \mathrm{~Hz}$, arom.-2', 6’), $7.00\left(2 \mathrm{H}, \mathrm{d},{ }^{3} J_{\mathrm{HH}}=8.3 \mathrm{~Hz}\right.$, arom.-2”, 6”), $6.99\left(1 \mathrm{H}, \mathrm{dd},{ }^{4} J_{\mathrm{PH}}=8.5\right.$ $\mathrm{Hz},{ }^{3} J_{\mathrm{HH}}=5.8 \mathrm{~Hz}$, quinone-3), $6.96\left(2 \mathrm{H}, \mathrm{d},{ }^{3} J_{\mathrm{HH}}=8.3 \mathrm{~Hz}\right.$, arom.- 3 ", 5 "), $6.78\left(1 \mathrm{H}, \mathrm{dd},{ }^{4} J_{\mathrm{PH}}\right.$ $=4.2 \mathrm{~Hz},{ }^{3} J_{\mathrm{HH}}=5.7 \mathrm{~Hz}$, quinone-4), $2.30\left(3 \mathrm{H}, \mathrm{s}, \mathrm{C} 4{ }^{\prime}-\mathrm{CH}_{3}\right), 2.23\left(3 \mathrm{H}, \mathrm{s}, \mathrm{C}-4\right.$ " $\left.-\mathrm{CH}_{3}\right), 1.46$ $\left(18 \mathrm{H}\right.$, brs, $\left.o-\mathrm{C}\left(\mathrm{CH}_{3}\right)_{3}\right), 1.26\left(9 \mathrm{H}, \mathrm{s}, p-\mathrm{C}\left(\mathrm{CH}_{3}\right)_{3}\right) ;{ }^{13} \mathrm{C}\left\{{ }^{1} \mathrm{H}\right\} \mathrm{NMR}\left(125 \mathrm{MHz}, \mathrm{CD}_{2} \mathrm{Cl}_{2}\right) \delta=$ $182.75\left(\mathrm{~d}, J_{\mathrm{PC}}=48.6 \mathrm{~Hz}, \mathrm{P}=\mathrm{C}\right), 154.36\left(\mathrm{~s}, \mathrm{Mes}^{*}-o\right), 150.91\left(\mathrm{~s}, \mathrm{Mes}^{*}-p\right), 145.23\left(\mathrm{~d}, J_{\mathrm{PC}}=\right.$

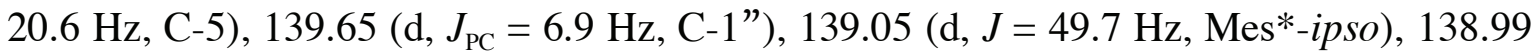
$\left(\mathrm{d}, J_{\mathrm{PC}}=6.3 \mathrm{~Hz}, \mathrm{C}-1\right.$ ') 137.92 (s, C-4”), 137.78 (s, C-4'), 137.77 (d, $\left.J_{\mathrm{PC}}=21.7 \mathrm{~Hz}, \mathrm{C}-3\right)$, $133.41\left(\mathrm{~d}, J_{\mathrm{PC}}=33.7 \mathrm{~Hz}, \mathrm{C}-4\right), 130.83\left(\mathrm{~d}, J_{\mathrm{PC}}=3.4 \mathrm{~Hz}, \mathrm{C}-2^{\prime}, 6^{\prime}\right), 130.33\left(\mathrm{~d}, J_{\mathrm{PC}}=36.0 \mathrm{~Hz}\right.$, C-6), 129.98 (d, $J_{\mathrm{PC}}=4.0 \mathrm{~Hz}, \mathrm{C}-2$ ", 6”), 129.17 (s, C-3', 5'), 129.06 (s, C-3”, 5”), 122.40 (s, Mes*-m), $38.30\left(\mathrm{~s}, \underline{\mathrm{C}}\left(\mathrm{CH}_{3}\right)_{3}-o\right), 35.26\left(\mathrm{~s}, \underline{\mathrm{C}}\left(\mathrm{CH}_{3}\right)_{3}-p\right), 32.79$ (d, $\left.J_{\mathrm{PC}}=6.3 \mathrm{~Hz}, \mathrm{C}\left(\mathrm{CH}_{3}\right)_{3}-o\right)$, 31.39 (s, $\left.\mathrm{C}\left(\mathrm{CH}_{3}\right)_{3}-p\right), 21.30$ (s, C- $\underline{C H}_{3}-4$ '), 21.25 (s, C- $\underline{\mathrm{CH}}_{3}-4$ "); ${ }^{31} \mathrm{P}$ NMR (81 MHz, $\left.\mathrm{CD}_{2} \mathrm{Cl}_{2}\right) \delta=198.9(\mathrm{~s}) ; \mathrm{IR}(\mathrm{KBr}) 2960(\mathrm{~s}), 2916(\mathrm{~m}), 2866(\mathrm{~m}), 1591(\mathrm{w}), 1508(\mathrm{~m}), 1458(\mathrm{~m})$, 1394(m), 1360(m), 1242(m), 1209(w), 1188(m), 1155(w), 1122(m), 1039(w), 1016(m), 877(w), 818(m), 783(m), 750(w), 719(w), 592(w), 490(w), and 447(w) $\mathrm{cm}^{-1}$; UV-Vis (hexanes) $\lambda_{\max } / \mathrm{nm}(\log \varepsilon) 449$ (4.60), 315 (sh; 3.79), 288 (4.19); LRMS (70 eV, EI) $\mathrm{m} / z$ (rel intensity) $552\left(M^{+} ; 100\right), 276\left(\mathrm{Mes}^{*} \mathrm{P}^{+}+1\right.$ and $\left.\mathrm{Tol}_{2} \mathrm{C}=\mathrm{C}_{4} \mathrm{H}_{2} \mathrm{~S}^{+}+1 ; 15\right)$; HRMS (70 eV, EI) Found $\mathrm{m} / z$ 552.3018. Calcd. for $\mathrm{C}_{37} \mathrm{H}_{45} \mathrm{PS}: M, 552.2980$. (E)-6b; ${ }^{31} \mathrm{P}$ NMR (81 MHz, $\left.\mathrm{C}_{6} \mathrm{D}_{6}\right) \delta=187.2(\mathrm{~s})$.

\section{Phosphathienoquinomethane (Z)-6c}

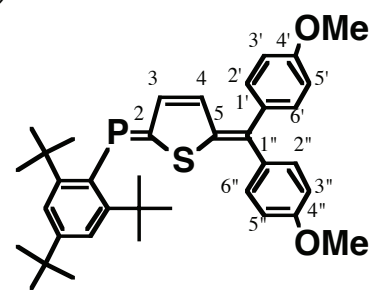


To a solution of 5-bromo-2-thienyl(2,4,6-tri-tert-butylphenyl)phosphine (8) (450 $\mathrm{mg}, 1.03 \mathrm{mmol})$ in ether $(15 \mathrm{~mL})$ was added $t$-butyllithium $(1.2 \mathrm{~mL}, 1.64 \mathrm{M}$ in pentane $)$ at $-78{ }^{\circ} \mathrm{C}$ and the mixture was stirred for $30 \mathrm{~min}$. The resultant orange solution was added to the colorless suspension of 4,4'-dimethoxybenzophenone ( $255 \mathrm{mg}, 1.05 \mathrm{mmol}$ ) in ether $(80 \mathrm{~mL})$ at $-78{ }^{\circ} \mathrm{C}$ and stirred for $30 \mathrm{~min}$. The mixture was gradually warmed to room temperature and stirred for $1 \mathrm{~h}$. The solution was washed with dilute $\mathrm{NH}_{4} \mathrm{Cl}$ solution and saturated $\mathrm{NaCl}$ solution. The organic layer was dried over anhydrous magnesium sulfate. After removal of the drying agent and the solvent, formation of the mixture of carbinol 10c and (Z)-6c was confirmed by ${ }^{31} \mathrm{P}$ NMR spectroscopy. The mixture of 10c and $(Z)-6 \mathbf{c}$ was dissolved in dry benzene $(15 \mathrm{~mL})$, added to a suspension of anhydrous copper(II) sulfate $(1.69 \mathrm{~g}, 8.69 \mathrm{mmol})$ in dry benzene $(10 \mathrm{~mL})$ at room temperature, and was refluxed for $3 \mathrm{~h}$. Consumption of the starting material and formation of $(Z)-6 \mathbf{c}$ were monitored by ${ }^{31} \mathrm{P}$ NMR spectroscopy. The reaction mixture was filtered to remove copper sulfate. After removal of the drying agent and the solvent, the residue was submitted to column chromatography (alumina, benzene) to give $514 \mathrm{mg}(0.878 \mathrm{mmol}$, $85 \%)$ of phosphathienoquinomethane (Z)-6c. 10c: ${ }^{31} \mathrm{P}$ NMR $\left(81 \mathrm{MHz}, \mathrm{C}_{6} \mathrm{D}_{6}\right) \delta=-76.4$ $\left(\mathrm{d}, J_{\mathrm{PH}}=232 \mathrm{~Hz}\right) . \quad(Z)-6 \mathrm{c}$ : orange crystals, $\mathrm{mp} 100.0-103.0{ }^{\circ} \mathrm{C}$ (Decomp.); ${ }^{1} \mathrm{H}$ NMR (500 $\left.\mathrm{MHz}, \mathrm{CD}_{2} \mathrm{Cl}_{2}\right) \delta=7.37\left(2 \mathrm{H}, \mathrm{d},{ }^{4} J_{\mathrm{PH}}=1.0 \mathrm{~Hz}, \mathrm{Mes}^{*}-m\right), 7.08\left(2 \mathrm{H}, \mathrm{d},{ }^{3} J_{\mathrm{HH}}=8.2 \mathrm{~Hz}\right.$, arom.$\left.3^{\prime}, 5^{\prime}\right), 7.06\left(2 \mathrm{H}, \mathrm{d},{ }^{3} J_{\mathrm{HH}}=8.4 \mathrm{~Hz}\right.$, arom.-2”, 6”), $7.05\left(2 \mathrm{H}, \mathrm{d},{ }^{3} J_{\mathrm{HH}}=8.5 \mathrm{~Hz}\right.$, arom. -2 ', 6’), $6.98\left(1 \mathrm{H}, \mathrm{dd},{ }^{4} J_{\mathrm{PH}}=8.3 \mathrm{~Hz},{ }^{3} J_{\mathrm{HH}}=5.8 \mathrm{~Hz}\right.$, quinone-3), $6.76\left(1 \mathrm{H}, \mathrm{dd},{ }^{4} J_{\mathrm{PH}}=4.3 \mathrm{~Hz},{ }^{3} J_{\mathrm{HH}}=\right.$ $5.8 \mathrm{~Hz}$, quinone-4), $6.68\left(2 \mathrm{H}, \mathrm{d},{ }^{3} J_{\mathrm{HH}}=8.9 \mathrm{~Hz}\right.$, arom.-3", 5”), $3.76\left(3 \mathrm{H}, \mathrm{s}, 4^{\prime}-\mathrm{OCH}_{3}\right), 3.71$ $\left(3 \mathrm{H}, \mathrm{s}, 4\right.$ " $\left.-\mathrm{OCH}_{3}\right), 1.47\left(18 \mathrm{H}\right.$, brs, $\left.o-\mathrm{C}\left(\mathrm{CH}_{3}\right)_{3}\right), 1.27\left(9 \mathrm{H}, \mathrm{s}, p-\mathrm{C}\left(\mathrm{CH}_{3}\right)_{3}\right) ;{ }^{13} \mathrm{C}\left\{{ }^{1} \mathrm{H}\right\} \mathrm{NMR}(125$ $\left.\mathrm{MHz}, \mathrm{CD}_{2} \mathrm{Cl}_{2}\right) \delta=182.81$ (d, $J_{\mathrm{PC}}=48.6 \mathrm{~Hz}, \mathrm{P}=\mathrm{C}$ ), 159.57 (s, C-4”), 159.38 (s, C-4'), $154.34\left(\mathrm{~s}, \mathrm{Mes}^{*}-o\right), 150.85$ (s, Mes*-p), $144.41\left(\mathrm{~d}, J_{\mathrm{PC}}=20.6 \mathrm{~Hz}, \mathrm{C}-5\right), 139.20\left(\mathrm{~d}, J_{\mathrm{PC}}=\right.$ 49.2 Hz, Mes*-ipso), 137.40 (d, $\left.J_{\mathrm{PC}}=21.7 \mathrm{~Hz}, \mathrm{C}-3\right), 135.03$ (d, $J_{\mathrm{PC}}=7.4 \mathrm{~Hz}, \mathrm{C}-1$ ”), 134.40 $\left(\mathrm{d}, J_{\mathrm{PC}}=5.7 \mathrm{~Hz}, \mathrm{C}-1^{\prime}\right), 133.54\left(\mathrm{~d}, J_{\mathrm{PC}}=33.1 \mathrm{~Hz}, \mathrm{C}-4\right), 132.24\left(\mathrm{~d}, J_{\mathrm{PC}}=3.4 \mathrm{~Hz}, \mathrm{C}-2\right.$ ', 6'), 131.53 (d, $J_{\mathrm{PC}}=3.4 \mathrm{~Hz}, \mathrm{C}-2$ ", 6”), 129.83 (d, $\left.J_{\mathrm{PC}}=35.4 \mathrm{~Hz}, \mathrm{C}-6\right), 122.40$ (s, Mes*-m), 113.89 (s, C-3',5'), 113.71 (s, C-3”, 5”), 55.65 (s, 4'-- $\left.\mathrm{OCH}_{3}\right), 55.52$ (s, 4”- $\left.\mathrm{OCH}_{3}\right), 38.33$ (s, $\left.\mathrm{C}\left(\mathrm{CH}_{3}\right)_{3}-o\right), 35.30\left(\mathrm{~s}, \underline{\mathrm{C}}\left(\mathrm{CH}_{3}\right)_{3}-p\right), 32.80\left(\mathrm{~d}, J_{\mathrm{PC}}=6.3 \mathrm{~Hz}, \mathrm{C}\left(\mathrm{CH}_{3}\right)_{3}-o\right), 31.49\left(\mathrm{~s}, \mathrm{C}\left(\mathrm{CH}_{3}\right)_{3}-p\right)$; ${ }^{31} \mathrm{P}$ NMR (81 MHz, $\left.\mathrm{CD}_{2} \mathrm{Cl}_{2}\right) \delta=196.3(\mathrm{~s}) ; \mathrm{IR}(\mathrm{KBr}) 2956(\mathrm{~s}), 2904(\mathrm{~m}), 2870(\mathrm{w}), 2833(\mathrm{w})$, 1601(m), 1506(m), 1460(m), 1394(w), 1360(w), 1308(w), 1284(w), 1250(s), 1176(w), 1097(m), 1034(s), 876(w), 856(w), 802(s), 752(w), 694(w), 596(w), 563(w), 405(w) cm ${ }^{-1}$; UV-Vis (hexanes) $\lambda_{\max } / \mathrm{nm}(\log \varepsilon) 456$ (4.60), 296 (4.24); LRMS (70 eV, EI) $\mathrm{m} / z$ (rel intensity) $584\left(M^{+} ; 100\right), 569\left(M^{+}-\mathrm{Me} ; 11\right)$; HRMS (70 eV, EI) Found $m / z$ 584.2879. Calcd. for $\mathrm{C}_{37} \mathrm{H}_{45} \mathrm{O}_{2} \mathrm{PS}: M, 584.2878$. (E)-6c: ${ }^{31} \mathrm{P}$ NMR (81 MHz, $\left.\mathrm{C}_{6} \mathrm{D}_{6}\right) \delta=184.2$ (s)

\section{$X$-ray crystallographic analysis of phosphathienoquinone $(Z)-6 b$}

Crystal data of (Z)-6b: $\mathrm{C}_{37} \mathrm{H}_{45} \mathrm{SP}, M=552.80$, orange prisms grown from diethyl ether, crystal dimensions $0.40 \times 0.25 \times 0.20 \mathrm{~mm}^{3}$, monoclinic, space group $P 2_{1} / \mathrm{n}$ (no. 14), $a=10.595(6), b=18.095(4), c=16.820(3) \AA, \beta=96.04(2)^{\circ}, V=3206(1) \AA^{3}, D_{\mathrm{c}}=1.145$ $\mathrm{gcm}^{-3}, \mu=0.174 \mathrm{~mm}^{-1}, T=115(1) \mathrm{K}, Z=4, F(000)=1192.00$. A Rigaku RAXIS-IV imaging plate area detector with graphite-monochromated MoK $\alpha$ radiation $(\lambda=0.71070$ $\AA$ ) was used. A symmetry-related absorption correction using the program ABSCOR was applied. Of 6060 reflections measured $\left(2 \theta_{\max }=51.0^{\circ}\right), 5866$ were observed $[I>0.0 \sigma(I)]$. The structure was solved by heavy-atom Patterson methods (PATTY), expanded using Fourier techniques (DIRDIF94), and refined by full-matrix least-squares of $F$ for 533 variable parameters. The non-hydrogen atoms were refined anisotropically, and hydrogen atoms isotropically. Goodness of fit $S=1.34$ for observed reflections, and $R_{1}=$ 0.035 for $I>2.0 \circ(I), R=0.042, R_{W}=0.048$ for all reflections. The maximum and 
minimum peaks on the final difference Fourier map corresponded to 0.32 and $-0.34 \mathrm{e}^{-3}$, respectively. Structure solution, refinement, and graphical representation were carried out using the teXsan package. CCDC 260774.

\section{Cyclic voltammetry}

Cyclic voltammetry was performed on a BAS CV-50W controller with a glassy carbon, Pt wire, and $\mathrm{Ag} / 0.01 \mathrm{M} \mathrm{AgNO} / 0.10 \mathrm{Mn}-\mathrm{Bu}_{4} \mathrm{NClO}_{4} / \mathrm{CH}_{3} \mathrm{CN}$ as a working, counter, and reference electrode, respectively $\left(E_{1 / 2}(\right.$ Ferrocene/Ferricinium $\left.)=0.18 \mathrm{~V}\right)$. $\mathrm{A}$ substrate ca. $10^{-4} \mathrm{M}$ was dissolved in tetrahydrofuran with $0.10 \mathrm{M} n-\mathrm{Bu}_{4} \mathrm{NClO}_{4}$ as a supporting electrolyte, and the solution was degassed by bubbling with nitrogen gas and measured under nitrogen at room temperature or $-78{ }^{\circ} \mathrm{C}$ with dry ice-acetone bath.

Figure 1. Cyclic voltammograms of 5 (a) at $296 \mathrm{~K}$, (b) at $195 \mathrm{~K}$, (c) (Z)-6a, (d) (Z)-6b, and (e) (Z)-6c at $296 \mathrm{~K}$ in tetrahydrofuran with $0.1 \mathrm{M} \mathrm{n}$ - $\mathrm{Bu}_{4} \mathrm{NClO}_{4}$. Scan rate: $30 \mathrm{mVs}^{-1}$.

(a) $296 \mathrm{~K}$
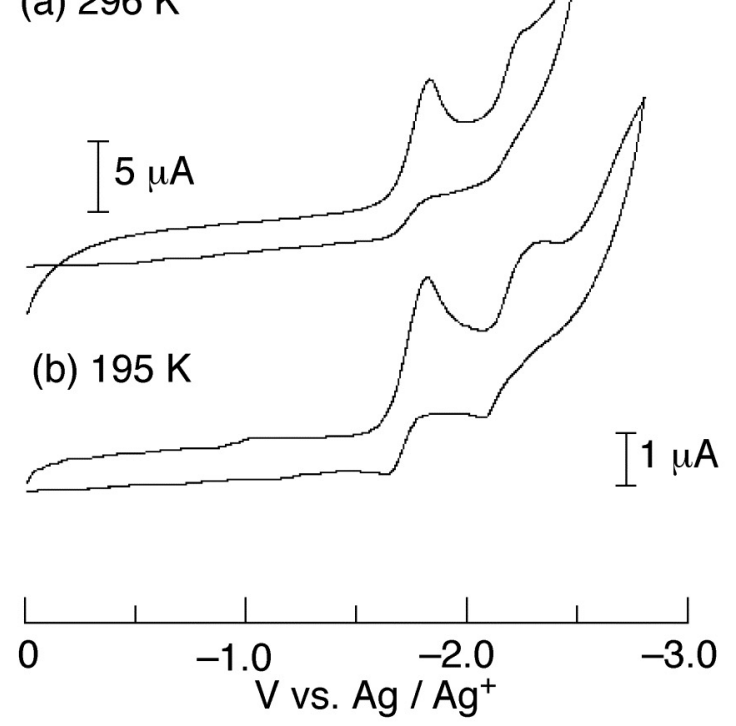

(c)

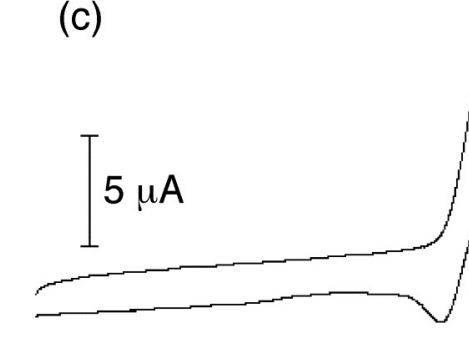

(d)

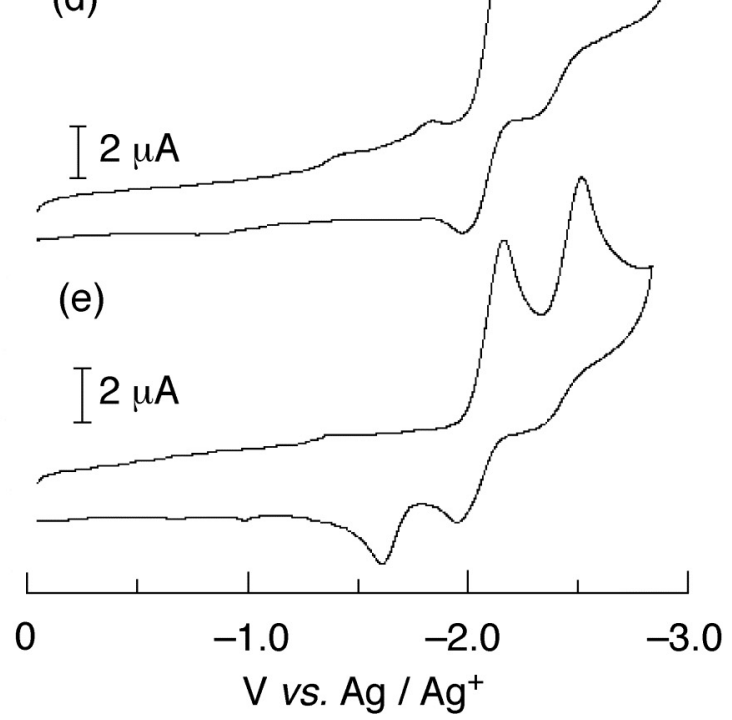

\section{EPR measurement}

X-band EPR spectra were measured on a Bruker ESP300E spectrometer at room temperature and $77 \mathrm{~K}$. X-band EPR spectra at $77 \mathrm{~K}$ were measured with a JEOL ESUCD2X liquid nitrogen Dewar. Tetrahydrofuran was distilled from sodium benzophenone ketyls just prior to use and transferred to a sample tube by bulb-to-bulb distillation. Samples were degassed by the freeze-and-thaw methods three times and allowed to react with alkaline metal mirror, which was distilled two times in the vacuum line. 
Figure 2. EPR spectra obtained after sodium reduction of 5 (a) at $293 \mathrm{~K}\left(g=2.005, a\left({ }^{31} \mathrm{P}\right)\right.$ $=5.7 \mathrm{mT}),(\mathrm{b}) 77 \mathrm{~K}\left(g_{\perp}=2.006, a_{\perp}\left({ }^{31} \mathrm{P}\right)=0.6 \mathrm{mT}, g_{/ /}=2.002, a_{/}\left({ }^{31} \mathrm{P}\right)=15.3 \mathrm{mT}\right.$, af $=5.5$ $\mathrm{mT}, \rho(\mathrm{P} 3 \mathrm{~s})=1 \%, a \mathrm{p}=4.9 \mathrm{mT}, \rho(\mathrm{P} 3 \mathrm{p})=37 \%)$, and $5{ }^{13} \mathrm{C}$, (c) at $293 \mathrm{~K}\left(g=2.005, a\left({ }^{31} \mathrm{P}\right)=\right.$ $\left.5.6 \mathrm{mT}, a\left({ }^{13} \mathrm{C}\right)=1.1 \mathrm{mT}\right),(\mathrm{d}) 77 \mathrm{~K}\left(g_{\perp}=2.006, a_{\perp}\left({ }^{31} \mathrm{P}\right)=0.8 \mathrm{mT}, g_{/ /}=2.002, a_{/ /}\left({ }^{31} \mathrm{P}\right)=15.3\right.$ $\mathrm{mT}, a_{\perp}\left({ }^{13} \mathrm{C}\right)=0.7 \mathrm{mT}, a_{/ /}\left({ }^{13} \mathrm{C}\right)=1.9 \mathrm{mT}, a \mathrm{f}\left({ }^{31} \mathrm{P}\right)=5.6 \mathrm{mT}, \rho(\mathrm{P} 3 \mathrm{~s})=1 \%, a \mathrm{p}\left({ }^{31} \mathrm{P}\right)=4.8 \mathrm{mT}$, $\left.\rho(\mathrm{P} 3 \mathrm{p})=37 \%, a \mathrm{f}\left({ }^{13} \mathrm{C}\right)=1.1 \mathrm{mT}, \rho(\mathrm{C} 2 \mathrm{~s})=2 \%, a \mathrm{p}\left({ }^{13} \mathrm{C}\right)=0.4 \mathrm{mT}, \rho(\mathrm{C} 2 \mathrm{p})=10 \%\right)$ in THF.
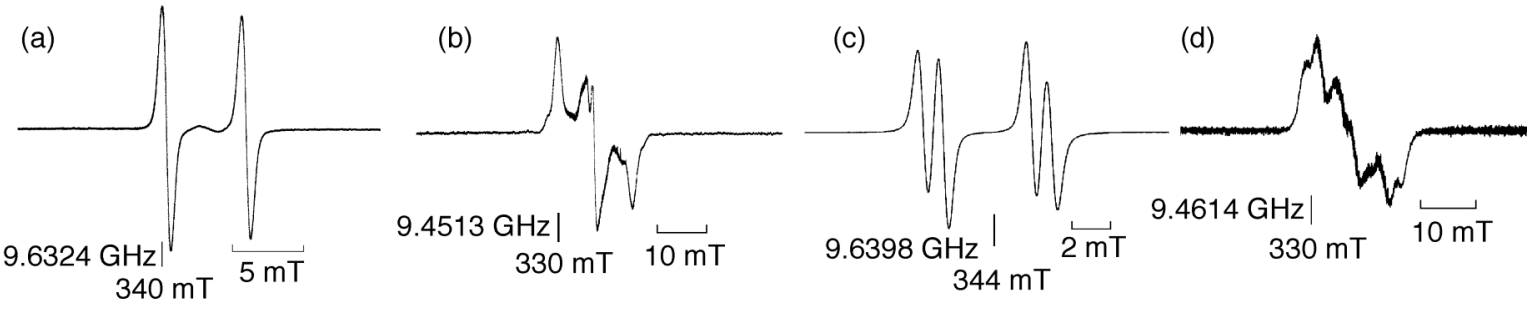

Figure 3. EPR spectra obtained after sodium reduction of (Z)-6a (a) at $293 \mathrm{~K}(g=2.004$, $\left.a\left({ }^{31} \mathrm{P}\right)=5.2 \mathrm{mT}\right),(\mathrm{b}) 77 \mathrm{~K}\left(g_{\perp}=2.006, a_{\perp}\left({ }^{31} \mathrm{P}\right)=0.2 \mathrm{mT}, g_{/ /}=2.002, a_{/ /}\left({ }^{31} \mathrm{P}\right)=13.8 \mathrm{mT}\right.$, af $=4.7 \mathrm{mT}, \rho(\mathrm{P} 3 \mathrm{~s})=1 \%, a \mathrm{p}=4.5 \mathrm{mT}, \rho(\mathrm{P} 3 \mathrm{p})=34 \%)$, and $(Z)-6 \mathrm{a}^{13}{ }^{13} \mathrm{C}(\mathrm{c})$ at $293 \mathrm{~K}(\mathrm{~g}=$ 2.004, $\left.a\left({ }^{31} \mathrm{P}\right)=5.2 \mathrm{mT}, a\left({ }^{13} \mathrm{C}\right)=1.0 \mathrm{mT}\right),(\mathrm{d}) 77 \mathrm{~K}\left(g_{\perp}=2.006, a_{\perp}\left({ }^{31} \mathrm{P}\right)=0.8 \mathrm{mT}, g_{/ /}=\right.$ 2.003, $a_{/ /}\left({ }^{31} \mathrm{P}\right)=14.0 \mathrm{mT}, a_{\perp}\left({ }^{13} \mathrm{C}\right)=0.6 \mathrm{mT}, a_{/ /}\left({ }^{13} \mathrm{C}\right)=1.9 \mathrm{mT}, a \mathrm{f}\left({ }^{31} \mathrm{P}\right)=5.2 \mathrm{mT}, \rho(\mathrm{P} 3 \mathrm{~s})=$ $1 \%, a \mathrm{p}\left({ }^{31} \mathrm{P}\right)=4.4 \mathrm{mT}, \rho(\mathrm{P} 3 \mathrm{p})=34 \%, a \mathrm{f}\left({ }^{13} \mathrm{C}\right)=1.0 \mathrm{mT}, \rho(\mathrm{C} 2 \mathrm{~s})=2 \%, a \mathrm{p}\left({ }^{13} \mathrm{C}\right)=0.4 \mathrm{mT}$, $\rho(\mathrm{C} 2 \mathrm{p})=11 \%)$ in THF.
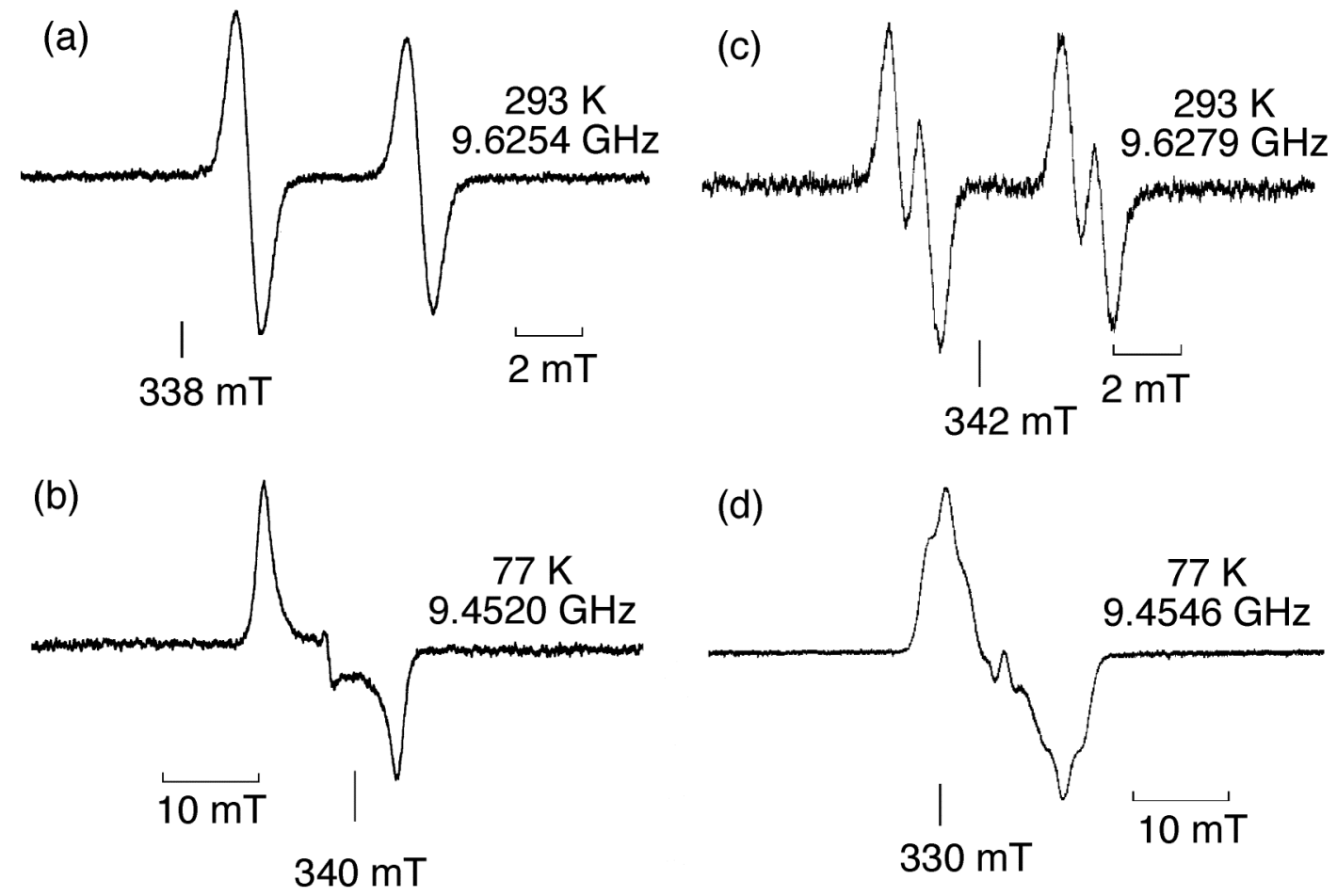
Figure 4. EPR spectra obtained after sodium reduction of (Z)-6b (a) at $293 \mathrm{~K}(g=2.0042$, $\left.a\left({ }^{31} \mathrm{P}\right)=4.9 \mathrm{mT}\right),(\mathrm{b}) 77 \mathrm{~K}\left(g_{/ /}=2.0025, a_{/ /}\left({ }^{31} \mathrm{P}\right)=13.0 \mathrm{mT}, a \mathrm{f}=4.3 \mathrm{mT}, \rho(\mathrm{P} 3 \mathrm{~s})=1 \%, a \mathrm{p}=\right.$ $4.3 \mathrm{mT}, \rho(\mathrm{P} 3 \mathrm{p})=33 \%)$ in THF.
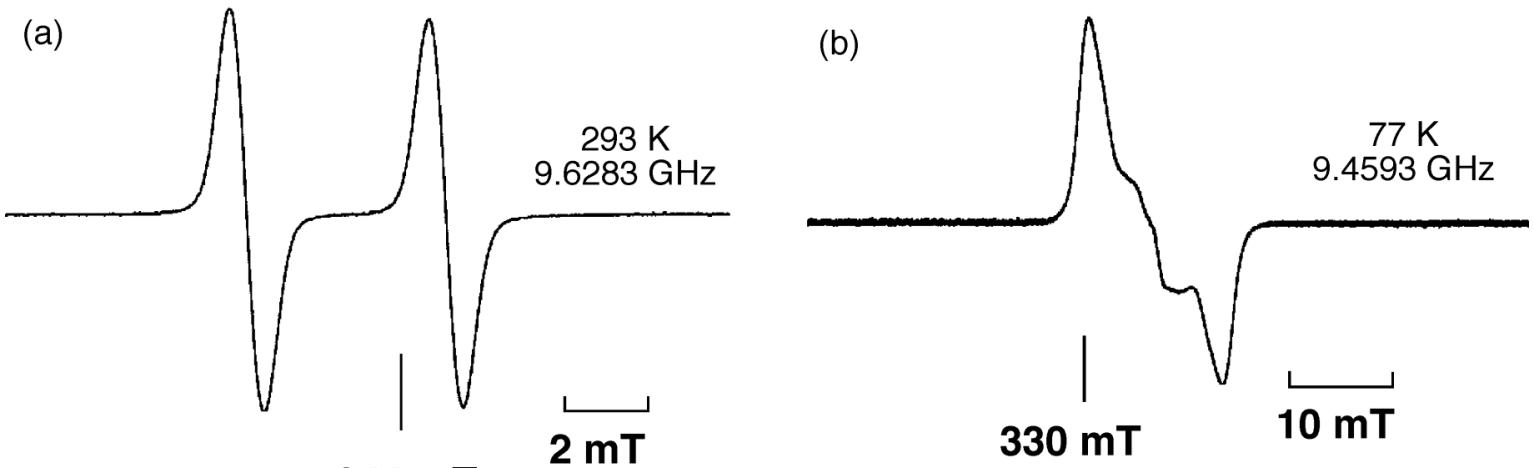

$344 \mathrm{mT}$

Figure 5. EPR spectra obtained after sodium reduction of (Z)-6c (a) at $293 \mathrm{~K}$ ( $g=2.0040$, $\left.a\left({ }^{31} \mathrm{P}\right)=4.6 \mathrm{mT}\right),(\mathrm{b}) 77 \mathrm{~K}\left(g_{/ /}=2.0029, a_{/ /}\left({ }^{31} \mathrm{P}\right)=12.5 \mathrm{mT}, a \mathrm{f}=4.2 \mathrm{mT}, \rho(\mathrm{P} 3 \mathrm{~s})=1 \%, a \mathrm{p}=\right.$ $4.2 \mathrm{mT}, \rho(\mathrm{P} 3 \mathrm{p})=32 \%)$ in $\mathrm{THF}$.

(a)

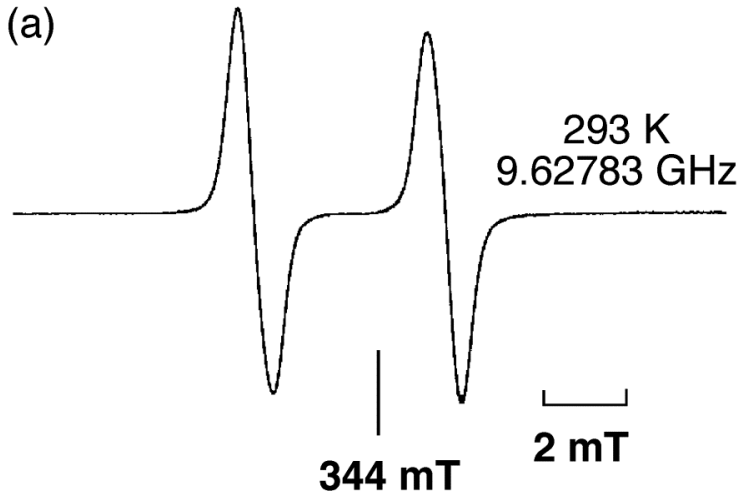

(b)

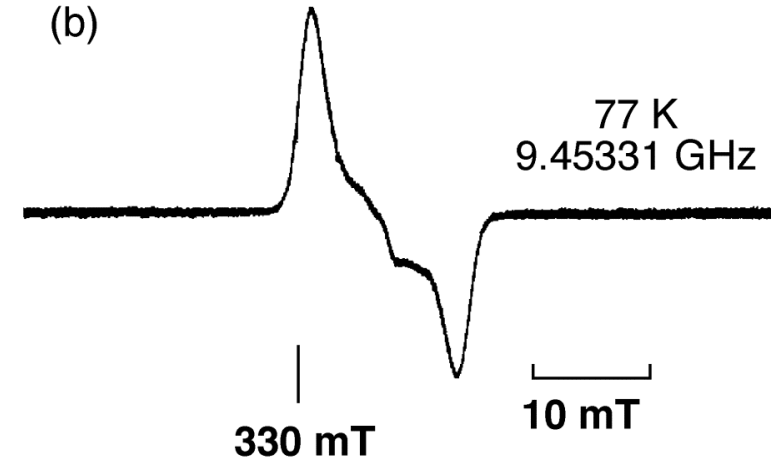

